\title{
Analytical Solutions for Side-branch Impedance Producing Spatial Localization of Acoustic Waves in Ducts with Varying Cross Sections
}

\author{
Yongxiong Xiao and Huancai Lu \\ Sound and Vibration Laboratory, National and International Joint Research Center for Special Purpose Equipment \\ and Advanced Processing Technology, College of Mechanical Engineering, Zhejiang University of Technology, 18 \\ Chaowang Road, Hangzhou 310014, China. E-mail: huancailu@zjut.edu.cn.
}

\section{Michael McFarland}

Sound and Vibration Laboratory, National and International Joint Research Center for Special Purpose Equipment and Advanced Processing Technology, College of Mechanical Engineering, Zhejiang University of Technology, 18 Chaowang Road, Hangzhou 310014, China.

Department of Aerospace Engineering, University of Illinois at Urbana-Champaign, 104 South Wright Street, Urbana, IL 61801, USA.

\section{Lawrence A. Bergman}

Department of Aerospace Engineering, University of Illinois at Urbana-Champaign, 104 South Wright Street, Urbana, IL 61801, USA.

\begin{abstract}
Daren Zhou
Sound and Vibration Laboratory, National and International Joint Research Center for Special Purpose Equipment and Advanced Processing Technology, College of Mechanical Engineering, Zhejiang University of Technology, 18 Chaowang Road, Hangzhou 310014, China.
\end{abstract}

\begin{abstract}
(Received 20 November 2020; accepted 23 April 2021)
Analytical mathematical models and solutions for spatial localization of acoustic waves through an impedance discontinuity produced by an intermediate damped side branch are studied in stationary media in ducts with varying cross sections. Three specific geometries, namely, with polynomial, sinusoidal, and exponential longitudinal variations, are investigated. The sound fields inside the ducts are modeled by Webster's horn equation. Travelingwave solutions are obtained by appropriate transformations. The side-branch impedances required for spatial localization (confinement) of traveling and standing waves are found analytically and verified numerically using three-dimensional finite element analysis. The impact of the longitudinal variation of the duct's cross-sectional area (CSA) on the side-branch impedance is examined. It was found that the required side-branch resistance changes more than the reactance with the variation of the duct CSA. A measure of a traveling wave is defined to quantitatively examine the spatial localization of acoustic waves. It was found that the CSA corrections on the side-branch impedances are important. The results of this study reveal the quantitative relationships between the side-branch impedance and the CSA variations for zero reflection from the impedance discontinuity. The mathematical approach presented is potentially helpful for a design of a full anechoic termination and energy localization in duct systems.
\end{abstract}

\section{INTRODUCTION AND MOTIVATION}

Side-branch acoustic resonators are conventionally used as band-stop filters in duct systems to stop the downstream transmission of acoustic waves. Recently, a nonresonant damped side branch has been used for spatial separation (localization) of acoustic traveling and standing waves in a duct with constant cross-sectional area (CSA), where the acoustic impedance of the side branch required to produce this localization is found analytically as a function of wavenumber and side-branch location. ${ }^{1-3}$ In structural dynamics, spatial separation of traveling and standing waves has also been studied for a non-dispersive taut string with an attached spring-dashpot support and a vibration absorber, ${ }^{4,5}$ a dispersive taut string on a partial viscoelastic foundation, ${ }^{6}$ and an Euler-Bernoulli beam with one or two spring-dashpot supports. ${ }^{7}$ The ducts, strings and beams stud- ied for localization of waves were all assumed to have constant geometries, as in the case of the duct ${ }^{1,2}$ shown in Fig. 1. However, ducts with varying CSA are commonly used in engineering and sometimes cannot be avoided. For example, the inlet and outlet ducts of ventilation systems usually have a varying CSA to accommodate technical design specifications. Anechoic treatment is required for those ducts. Acoustic energy is transmitted from the traveling-wave region to the standingwave region with minimum energy being reflected back to the sound source, creating an anechoic termination. The purpose of this work is to research whether exact analytical solutions for the required acoustic impedance of the side branch exist for localization of traveling and standing waves in ducts with varying CSA.

The control of acoustic traveling and standing waves prop- 


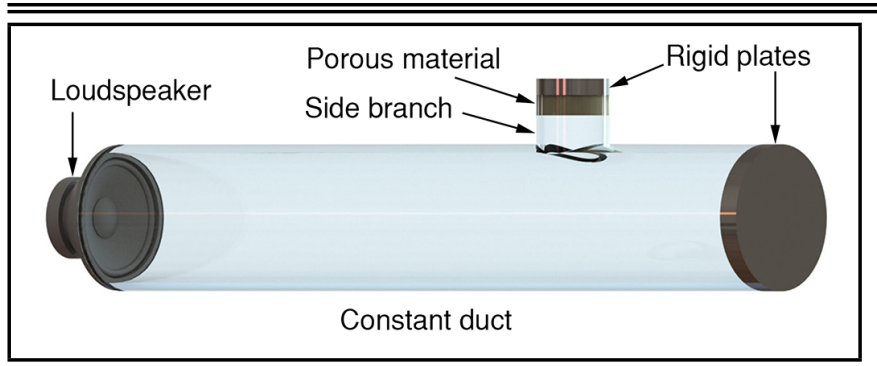

Figure 1. Schematic representation of a circular duct of constant CSA connected at a right angle to a nonresonant side branch with a piece of porous material.

agating in waveguides with a longitudinally varying crosssection has found many engineering applications. ${ }^{8-11}$ For lowfrequency acoustic waves propagating in ducts with slowly varying CSA, the governing equation of acoustic pressure is well approximated by the one-dimensional (1D) Webster's horn equation. ${ }^{12-15}$ Analytical solutions for wave propagation in ducts of conical and exponential shapes in the presence of low Mach numbers were derived by Easwaran and Munjal. ${ }^{16}$ Exact standing-wave solutions were obtained using appropriate transformations for a rod with polynomial or sinusoidal geometry by Kumar and Sujith. ${ }^{17}$ Approximate analytical solutions for ducts with axial temperature gradients with and without CSA variations and mean flow were presented by Cummings ${ }^{18}$ and Dokumaci. ${ }^{19}$ Exact travelling wave-type solutions are obtained for quasi-one-dimensional, transient acoustic wave propagation in ducts with a class of temperature and CSA variations by Subrahmanyam and Sujith. ${ }^{20}$ Compact nozzle solutions for quasi-one-dimensional linearized were obtained by Duran and Moreau by the Magnus expansion. ${ }^{21} \mathrm{~A}$ semi-analytical solution for acoustic wave propagation in ducts with arbitrary CSA variations and temperature gradient in the presence of non-isentropic mean flow is developed by Yeddula and Morgans. ${ }^{22}$ For waveguides with more general CSA variations, Webster's horn equation can be solved by numerical methods ${ }^{23,24}$ or perturbation methods. ${ }^{25}$ In this work, we build on these existing solutions for ducts with a polynomial, sinusoidal or exponential variation in the longitudinal direction, with the important difference that we focus on deriving exact solutions for the acoustic impedance of the side-branch required for spatial localization of traveling and standing waves.

In the work to follow, the conditions for localization by a side branch of traveling and standing waves in ducts of polynomial, sinusoidal, and exponential variations in CSA are derived analytically. These conditions are subsequently verified numerically using three-dimensional (3D) finite element analysis (FEA). The effects of variation in CSA parameters on the required acoustic impedance of the side branch are studied. A measure of a traveling wave is defined, and it is evaluated with respect to changes in the CSA parameters using 3D FEA.

\section{MATHEMATICAL MODELLING}

A low-frequency acoustic wave propagating in stationary perfect air within a rigid-walled duct of slowly varying CSA is approximated by the $1 \mathrm{D}$ Webster's horn equation. The assumptions of harmonic time dependence and separability, such that $p(x, t)=\hat{p}(x) e^{\mathrm{j} \Omega t}$, where $\mathrm{j}=\sqrt{-1}$, result in the relation: ${ }^{12}$

$$
\frac{\mathrm{d}^{2} \hat{p}(x)}{\mathrm{d} x^{2}}+\frac{1}{S(x)} \frac{\mathrm{d} S(x)}{\mathrm{d} x} \frac{\mathrm{d} \hat{p}(x)}{\mathrm{d} x}+k^{2} \hat{p}(x)=0 ;
$$

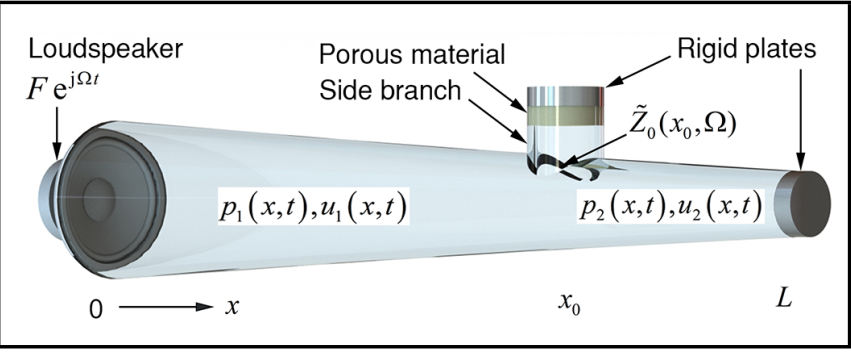

Figure 2. Circular duct with polynomial CSA variation connected at a right angle to an intermediate damped side branch; the CSA of the duct is $s(x)=$ $\left(a_{1} x+a_{2}\right)^{n}$, where $a_{1}=-0.15 \mathrm{~m}^{2 / n-1}, a_{2}=0.30 \mathrm{~m}^{2 / n}, n=3$, and $L=1 \mathrm{~m}$.

where $x$ is the longitudinal coordinate, $\hat{p}(x)$ is the complex acoustic pressure amplitude, $S(x)$ is the CSA distribution, $k=$ $\Omega / c_{0}$ is the wavenumber, $\Omega$ is the angular frequency, and $c_{0}$ is the adiabatic speed of sound.

In the following sections, necessary and sufficient conditions for localization of traveling and standing waves in rigid circular ducts with polynomial, sinusoidal and exponential variations in CSA are derived in closed form.

\subsection{Polynomial Area Variation}

The mathematical expression for the polynomial variation of CSA is:

$$
S(x)=\left(a_{1} x+a_{2}\right)^{n} ;
$$

where $n$ is limited to integers in this work. The parameters $a_{1}$, $a_{2}$, and $n$ should be chosen such that $S(x)>0,0 \leq x \leq L$, where $L$ is the total length of a duct. For a polynomial duct, $a_{1}$ controls the slope of the radius, while $a_{2}$ determines the initial radius. The dimensions of $a_{1}$ and $a_{2}$ are $m^{2 / n-1}$ and $m^{2 / n}$, respectively. A side branch connects at a right angle to the main duct, and a plug of acoustic material is inserted at the closed end of the side branch to tune its input impedance. Both the end of the side branch and the main duct are terminated with rigid discs. Figure 2 is a three-dimensional rendering of such a system, showing a rigid circular duct with polynomial CSA variation connected to an intermediate side-branch.

The analytical solutions can be found in a references. ${ }^{17}$ For the case of $\nu$ integer, the solution can be expressed in the form of a traveling wave by:

$$
y=C_{1} H_{\nu}^{(1)}(z)+C_{2} H_{\nu}^{(2)}(z)
$$

where $H_{\nu}^{(1)}(z)=J_{\nu}(z)-\mathrm{j} Y_{\nu}(z)$ is the Hankel function of the first kind representing a forward-propagating wave, and $H_{\nu}^{(2)}(z)=J_{\nu}(z)+\mathrm{j} Y_{\nu}(z)$ is the Hankel function of the second kind representing a backward-propagating wave; $c_{1}$ and $c_{2}$ are again two arbitrary constant coefficients.

The acoustic pressures in the two regions of the duct, to the left and right of the side branch, are, respectively:

$$
\begin{array}{r}
p_{1}(z, t)=S^{\alpha}\left[C_{1} H_{\nu}^{(1)}(z)+C_{2} H_{\nu}^{(2)}(z)\right] e^{\mathrm{j} \Omega t} \\
k a_{2} a_{1} \leq z \leq z_{0} ;
\end{array}
$$

$$
p_{2}(z, t)=S^{\alpha}\left[D_{1} H_{\nu}^{(1)}(z)+D_{2} H_{\nu}^{(2)}(z)\right] e^{\mathrm{j} \Omega t},
$$

$$
z_{0} \leq z \leq z_{L} .
$$


Here, $z=\lambda S^{\beta}=k\left(a_{1} x+a_{2}\right) / a_{1}, z_{0}=k\left(a_{1} x_{0}+a_{2}\right) a_{1}$, $z_{L}=k\left(a_{1} L+a_{2}\right) / a_{1}$, and $x_{0}$ is the axial position of the side branch. Similarly, the acoustic particle velocities $u_{1}(x, t)$ and $u_{2}(x, t)$ in the two regions of the duct are determined by Euler's equation:

$$
\frac{\partial}{\partial t} u_{i}(x, t)=-\frac{1}{\rho_{0}} \frac{\partial}{\partial x} p_{i}(x, t), \quad i=1,2 ;
$$

where $\rho_{0}$ is the ambient air density. The boundary conditions at the left and right ends of the duct can be written as:

$$
\begin{gathered}
p_{1}(x=0, t)=F e^{\mathrm{j} \Omega t} ; \\
u_{2}(x=L, t)=0 .
\end{gathered}
$$

Assuming that the diameter of the side branch is much small than a wavelength, the acoustic pressure is continuous across the side-branch:

$$
p_{1}\left(x_{0}, t\right)=p_{2}\left(x_{0}, t\right) .
$$

The conservation of volume velocity at the position of the sidebranch requires:

$$
S\left(x_{0}\right) u_{1}\left(x_{0}, t\right)=S\left(x_{0}\right) u_{2}\left(x_{0}, t\right)+S_{b} u_{b}\left(x_{0}, t\right) .
$$

Dividing Eq. (10) by Eq. (9) and taking the complex amplitudes gives:

$$
\frac{\hat{u}_{1}\left(x_{0}, \Omega\right)-\hat{u}_{2}\left(x_{0}, \Omega\right)}{\hat{p}_{1}\left(x_{0}, \Omega\right)}=\frac{S_{b}}{S\left(x_{0}\right)} \frac{1}{\tilde{Z}_{0}\left(x_{0}, \Omega\right)} ;
$$

where $\tilde{Z}_{0}\left(x_{0}, \Omega\right)$ is the specific acoustic impedance of the side branch, defined as the ratio of the complex amplitudes of the acoustic pressure and particle velocity at the inlet of the side branch; $S_{b}$ is the CSA of the side branch; and $F$ is a constant representing the amplitude of the excitation. The expressions for $C_{1}, C_{2}, D_{1}$, and $D_{2}$ are obtained from Eqs. (7)-(11) as:

$$
\begin{gathered}
C_{1}=\frac{F a_{2}^{-v}}{G_{1}}\left(H_{\nu-1}^{(2)}\left(z_{L}\right)\left(\pi z_{0} H_{\nu}^{(1)}\left(z_{0}\right) H_{\nu}^{(2)}\left(z_{0}\right)+4 Z_{0}\right)\right. \\
\left.-\pi z_{0} H_{\nu-1}^{(1)}\left(z_{L}\right)\left(H_{\nu}^{(2)}\left(z_{0}\right)\right)^{2}\right) ; \\
C_{2}=\frac{F a_{2}^{-v}}{G_{1}}\left(H_{\nu-1}^{(1)}\left(z_{L}\right)\left(\pi z_{0} H_{\nu}^{(1)}\left(z_{0}\right) H_{\nu}^{(2)}\left(z_{0}\right)-4 Z_{0}\right)\right. \\
\left.-\pi z_{0} H_{\nu-1}^{(2)}\left(z_{L}\right)\left(H_{\nu}^{(1)}\left(z_{0}\right)\right)^{2}\right) ; \\
D_{1}=\frac{4 F}{G_{1}} a_{2}^{-v} Z_{0} H_{\nu-1}^{(2)}\left(z_{L}\right) ; \\
D_{2}=-\frac{4 F}{G_{1}} a_{2}^{-v} Z_{0} H_{\nu-1}^{(1)}\left(z_{L}\right) ;
\end{gathered}
$$

where $Z_{0}=S_{b} \tilde{Z}_{0}\left(\rho_{0} c_{0} S\left(x_{0}\right)\right)$ is the normalized acoustic impedance of the side-branch and:

$$
\begin{gathered}
G_{1}= \\
H_{\nu}^{(2)}\left(\frac{a_{2} k}{a_{1}}\right)\left(H_{\nu-1}^{(1)}\left(z_{L}\right)\left(\pi z_{0} H_{\nu}^{(1)}\left(z_{0}\right) H_{\nu}^{(2)}\left(z_{0}\right)-4 Z_{0}\right)\right. \\
\left.-\pi z_{0}\left(H_{\nu}^{(1)}\left(z_{0}\right)\right)^{2} H_{\nu-1}^{(2)}\left(z_{L}\right)\right) \\
+H_{\nu}^{(1)}\left(\frac{a_{2} k}{a_{1}}\right)\left(H_{\nu-1}^{(2)}\left(z_{L}\right)\left(\pi z_{0} H_{\nu}^{(1)}\left(z_{0}\right) H_{\nu}^{(2)}\left(z_{0}\right)+4 Z_{0}\right)\right. \\
\left.-\pi z_{0}\left(H_{\nu}^{(2)}\left(z_{0}\right)\right)^{2} H_{\nu-1}^{(1)}\left(z_{L}\right)\right) .
\end{gathered}
$$

The side-branch impedance for localization of the traveling and standing waves is determined by setting to zero the coefficient of the wave reflected from the branch back to the source, ${ }^{1}$ i.e., setting $C_{2}=0$, giving:

$$
\begin{aligned}
& Z_{0}= \\
& \frac{1}{4} \pi z_{0} H_{\nu}^{(1)}\left(z_{0}\right)\left(H_{\nu}^{(2)}\left(z_{0}\right)-\frac{H_{\nu}^{(1)}\left(z_{0}\right) H_{\nu-1}^{(2)}\left(z_{L}\right)}{H_{\nu-1}^{(1)}\left(z_{L}\right)}\right) .
\end{aligned}
$$

Equation (17) has a singularity at $a_{1}=0$, which means it cannot be used for ducts with a constant CSA. The real part of Eq. (17) can be simplified to:

$$
\begin{aligned}
& \operatorname{Re}\left(Z_{0}\right)= \\
& \frac{\pi z_{0}}{2} \frac{\left(Y_{v}\left(Z_{0}\right) J_{v-1}\left(Z_{L}\right)-J_{v}\left(Z_{0}\right) Y_{v-1}\left(Z_{L}\right)\right)^{2}}{J_{v-1}^{2}\left(Z_{L}\right)+Y_{v-1}^{2}\left(Z_{L}\right)} \geq 0
\end{aligned}
$$

which is consistent with its physical interpretation as a local source of passive damping.

Substituting Eq. (17) into Eqs. (12)-(16) gives:

$$
\begin{gathered}
C_{1}={\frac{F a_{2}^{-v}}{H} \nu}_{\nu}^{(1)}\left(a_{2} k a_{1}\right) ; \\
C_{2}=0
\end{gathered}
$$

$$
\begin{aligned}
& D_{1}=\frac{F a_{2}^{-v} H_{\nu}^{(1)}\left(z_{0}\right) H_{\nu-1}^{(2)}\left(z_{L}\right)}{-2 \mathrm{j} H_{\nu}^{(1)}\left(a_{2} k a_{1}\right) E_{1}} ; \\
& D_{2}=\frac{F a_{2}^{-v} H_{\nu}^{(1)}\left(z_{0}\right) H_{\nu-1}^{(1)}\left(z_{L}\right)}{2 \mathrm{j} H_{\nu}^{(1)}\left(a_{2} k a_{1}\right) E_{1}} ;
\end{aligned}
$$

where,

$$
E_{1}=J_{v-1}\left(z_{L}\right) Y_{v}\left(z_{0}\right)-J_{v}\left(z_{0}\right) Y_{v-1}\left(z_{L}\right) .
$$

Upon substituting Eqs. (19)-(23) into Eqs. (4)-(5), it is clear that only a forward-traveling wave exists in the left region of the duct because the coefficient of the reflected waves there is zero. However, caution must be used when $E_{1}=0$, $D_{1}=D_{2}=\infty$, conditions corresponding to resonance in the right region of duct. The existence of this singularity implies that Eq. (17) is a necessary, but not sufficient, condition for realization of a traveling wave in the left region of the duct. Thus, necessary and sufficient conditions are both Eq. (17) and the requirement that $E_{1} \neq 0$. In addition, for rigid-walled ducts of circular CSA, plane waves can propagate only if their frequency is sufficiently small that $\Omega<1.84 c_{0} / R$, where is $R=\max [r(x)]$ the maximum radius of the duct over its entire length. ${ }^{26}$

\subsection{Sinusoidal Area Variation}

The expression for sinusoidal variation of the CSA of the duct is: ${ }^{17}$

$$
S(x)=S(0) \sin ^{2}\left(b_{1} x+b_{2}\right) .
$$

Note that the parameters in Eq. (28) must be constrained so that the area of the duct does not approach zero along its length. The system is otherwise identical to that of the polynomial duct. An example of a rigid-walled circular duct with sinusoidal area variation is shown in Fig. 3. 


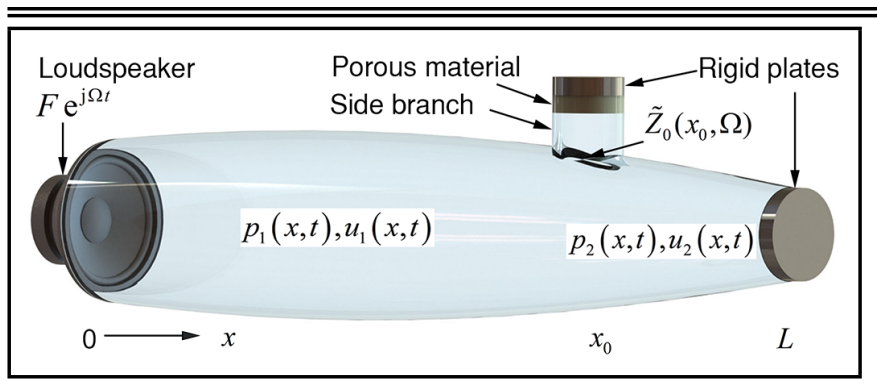

Figure 3. A circular duct with sinusoidal CSA variation connected at a right angle to an intermediate damped side branch; $S(x)=S(0) \sin ^{2}\left(b_{1} x+b_{2}\right)$, where $b_{1}=\pi / 2, b_{2}=\pi / 3, S(0)=0.0346 \mathrm{~m}^{2}$, and $L=1 \mathrm{~m}$.

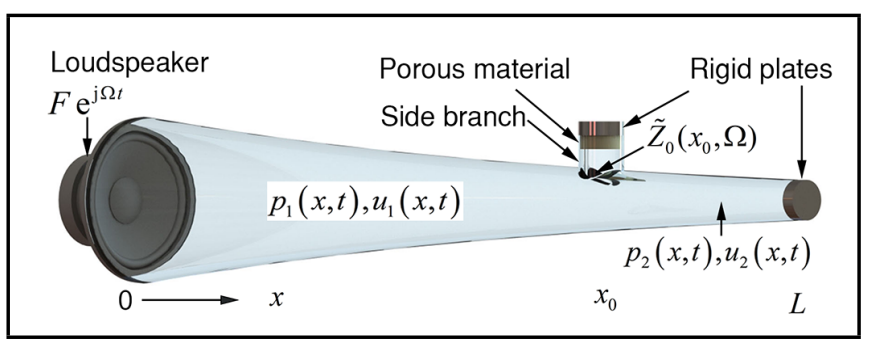

Figure 4. A circular duct with exponential CSA variation connected at a right angle to an intermediate damped side branch; $S(x)=S(0) e^{2 c_{1} x}$, where $S(0)=0.0254 \mathrm{~m}^{2}, c_{1}=-0.7$, and $L=1 \mathrm{~m}$.

The side-branch impedance producing localization of traveling and standing waves is obtained as (see Appendix A):

$$
Z_{0}=\frac{1}{2}+\frac{e^{2 \mathrm{j} k_{1}\left(L-x_{0}\right)}\left(k_{1}+\mathrm{j} b_{1} \cot \left(b_{1} L+b_{2}\right)\right)}{2\left(k_{1}-\mathrm{j} b_{1} \cot \left(b_{1} L+b_{2}\right)\right)} .
$$

Again, for passive damping $\operatorname{Re}\left(Z_{0}\right) \geq 0$, which is clearly the case in Eq. (25), as:

$$
\begin{aligned}
& \operatorname{Re}\left(Z_{0}\right)=\frac{\sin ^{2}\left(b_{1} L+b_{2}\right)}{4 k^{2} \sin ^{4}\left(b_{1} L+b_{2}\right)+b_{1}^{2} \sin ^{2}\left(2\left(b_{1} L+b_{2}\right)\right)} \\
& \times\left[\left(k_{1}-b_{1}\right) \sin \left(\left(b_{1}+k_{1}\right) L+b_{2}-k_{1} x_{0}\right)\right. \\
& \left.+\left(b_{1}+k_{1}\right) \sin \left(\left(b_{1}-k_{1}\right) L+b_{2}+k_{1} x_{0}\right)\right]^{2} \geq 0
\end{aligned}
$$

It is found that when $b_{1}=0$, Eq. (25) reduces to the conditions for localization of traveling and standing waves in a duct with a constant $\mathrm{CSA}^{1}$ :

$$
Z_{0}=\frac{1}{2}\left(1+\mathrm{e}^{2 \mathrm{j} k\left(L-x_{0}\right)}\right) .
$$

Necessary and sufficient conditions are Eq. (25) and $E_{2} \neq 0$ (see the Appendix A).

\subsection{Exponential Area Variation}

The expression for exponential variation of the CSA of the duct is:

$$
S(x)=S(0) e^{2 c_{1} x} .
$$

A system consisting of a rigid-walled circular duct with exponential area variation is shown in Fig. 4.

The side-branch impedance for realization of a traveling wave and standing waves is obtained as (see Appendix B):

$$
Z_{0}=k \frac{c_{1}-c_{1} \mathrm{e}^{2 \mathrm{j} k_{2}\left(L-x_{0}\right)}+\mathrm{j} k_{2}\left(1+\mathrm{e}^{2 \mathrm{j} k_{2}\left(L-x_{0}\right)}\right)}{2 k_{2}\left(\mathrm{j} k_{2}+c_{1}\right)} .
$$

As indicated earlier, passive damping requires $\operatorname{Re}\left(Z_{0}\right) \geq 0$, which is clearly the case in Eq. (29):

$$
\begin{aligned}
& \operatorname{Re}\left(Z_{0}\right)= \\
& \frac{e^{c_{1} x_{0}} k\left(c_{1} \sin \left[k_{2}\left(L-x_{0}\right)\right]-k_{2} \cos \left[k_{2}\left(L-x_{0}\right)\right]\right)^{2}}{k_{2}\left(c_{1}^{2}+k_{2}^{2}\right)} \geq 0 .
\end{aligned}
$$

When $c_{1}=0$ the duct becomes a uniform circular duct and Eq. (29) reduces to the condition for localization of traveling and standing waves in a duct with constant CSA, i.e., Eq. (27).

Necessary and sufficient conditions for localization in this case are Eq. (29) and $c_{1} \neq k_{2} \cot \left(k_{2}\left(L-x_{0}\right)\right.$ ) (see Appendix B).

\subsection{Traveling-Wave Measure}

In order to evaluate the conditions for realization of a traveling wave, a traveling-wave measure is defined. Since a traveling wave has a linear phase distribution in the longitudinal direction, the deviation of the phase of a wave from that of a traveling wave is defined as the traveling-wave measure $\varepsilon$ :

$$
\varepsilon=1-\frac{\left\|\phi_{\mathrm{w}}(x)-\phi_{\mathrm{t}}(x)\right\|_{2}}{\left\|\phi_{\mathrm{t}}(x)\right\|_{2}}, \quad 0<x<x_{0} ;
$$

where $\phi_{\mathrm{w}}(x)$ is the phase of the wave to be measured; $\phi_{\mathrm{t}}(x)=$ $-k x$ is the phase of an ideal traveling wave, which is a linear function of position with a slope of the wavenumber; and $\|\cdot\|_{2}$ is the 2-norm. Thus, $\varepsilon=1$ corresponds to an ideal traveling wave.

\section{NUMERICAL VERIFICATION}

The analytical solutions for side-branch impedances resulting in localization of traveling and standing waves are verified numerically in this section. The results from analytical solutions are compared with those from 3D FEA performed with ANSYS $^{\circledR}$. The reason for using 3D instead of $2 \mathrm{D}$ axisymmetric FEA is that the impedance discontinuity introduced by the damped side-branch is modeled by an impedance sheet, ${ }^{27}$ which is supported only by the 3D acoustic element (FLUID30). A volume-sweep method is used to create the meshes. ${ }^{28}$ A mesh size of $5 \mathrm{~mm}$ is specified. The total numbers of elements are 40400, 60092, 27600 for the polynomial, sinusoidal and exponential ducts, respectively. The FE meshes of these ducts are shown in Figs. 5(a), (b) and (c), where an impedance sheet normal to the duct axis has been used to represent an impedance discontinuity created by the side branch. The ducts are axisymmetric, so only a quarter of each model is used in order to reduce the computational effort.

The frequency of sound is set to $f=1000 \mathrm{~Hz}$, and the amplitude of the excitation is $F=1 \mathrm{~Pa}$. The length of the duct is $L=1 \mathrm{~m}$; the position of the side branch is chosen arbitrarily as $x_{0}=0.70 \mathrm{~m}$; and we take $S_{b}=S\left(x_{0}\right)$. The sound speed is $c_{0}=343 \mathrm{~m} / \mathrm{s}$. Other geometrical parameters of the polynominal, sinusoidal and exponential ducts are listed in Table 1 . The resulting duct geometries are depicted in Figs. 2, 3, and 4.

The normalized phases along the axial coordinate, obtained from the analytical solutions and 3D FEA for polynomial, sinusoidal and exponential ducts, are shown in Figs. 6(a), (b) and (c), respectively. The spatial phases are almost linear functions of the axial position in the left regions of the ducts, which means that traveling waves are realized there. On the other 
Table 1. Geometrical parameters and side-branch impedances for the numerical studies.

\begin{tabular}{|c|c|c|c|}
\hline & Polynominal duct & Sinusoidal duct & Exponential duct \\
\hline $\begin{array}{l}\text { Geometrical } \\
\text { parameters }\end{array}$ & $\begin{array}{c}a_{1}=-0.08 \mathrm{~m}^{2 / n-1} \\
a_{2}=-0.30 \mathrm{~m}^{2 / n} \\
n=3\end{array}$ & $\begin{array}{c}b_{1}=\pi / 2 \\
b_{2}=\pi / 3 \\
S(0)=0.0346 \mathrm{~m}^{2}\end{array}$ & $\begin{array}{c}S(0)=0.0254 \mathrm{~m}^{2} \\
C_{1}=-0.70\end{array}$ \\
\hline $\begin{array}{l}\text { Normalized side- } \\
\text { branch impedance } Z_{0}\end{array}$ & $0.4673-0.4990 \mathrm{~J}$ & $0.3724-0.4834 \mathrm{~J}$ & $0.4686-0.4992 \mathrm{~J}$ \\
\hline
\end{tabular}

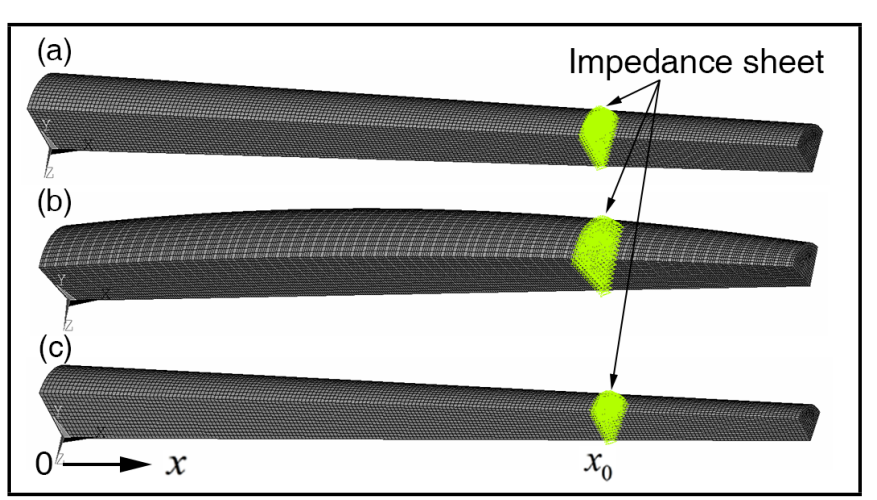

Figure 5. FE meshes of the (a) polynomial, (b) sinusoidal and (c) exponential ducts (a quarter of the model) using volume-sweep mesh method.

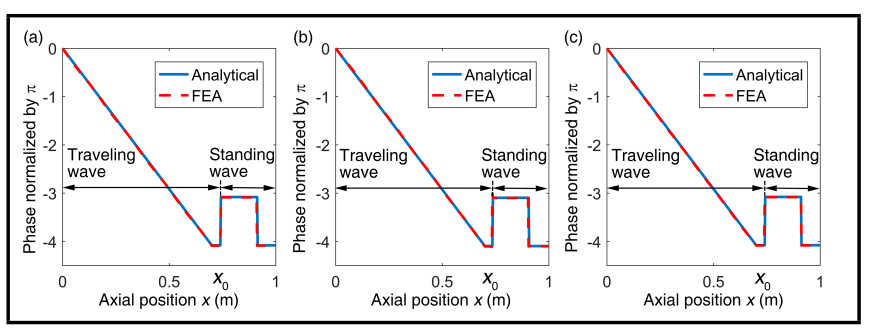

Figure 6. Normalized phase along the axis of the (a) polynomial (b) sinusoidal and (c) exponential ducts obtained from the analytical solutions (blue solid lines) and 3D FEA (red dashed lines).

hand, the phases are simply in- or out-of-phase in the right regions of the ducts, which means that standing waves occur. The acoustic pressure amplitudes obtained from the analytical solutions and 3D FEA for polynomial, sinusoidal and exponential ducts are shown in Figs. 7(a), (b) and (c), respectively.

The acoustic pressure amplitudes increase as the duct cross sections decrease. No acoustic pressure nodes exist in the left regions of the ducts, while two acoustic pressure nodes are present in the right regions. The discrepancies in the acoustic pressure amplitudes between the analytical solutions and 3D FEA indicate that there are some small reflections due to variation of acoustic impedance and 3D wave effects in the duct. ${ }^{20}$ Therefore, the verification of side-branch impedances for localization of traveling and standing waves is undertaken here in more detail.

The radii of the polynomial, sinusoidal and exponential ducts are plotted against the geometrical parameters in

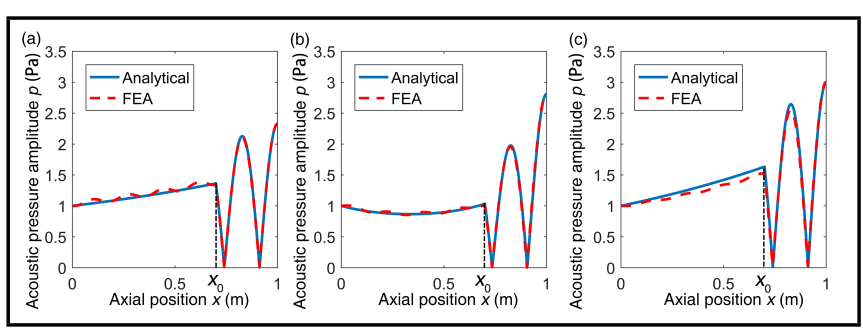

Figure 7. Acoustic pressure amplitude along the axis of the (a) polynomial (b) sinusoidal and (c) exponential ducts obtained from the analytical solutions (blue solid lines) and 3D FEA (red dashed lines).

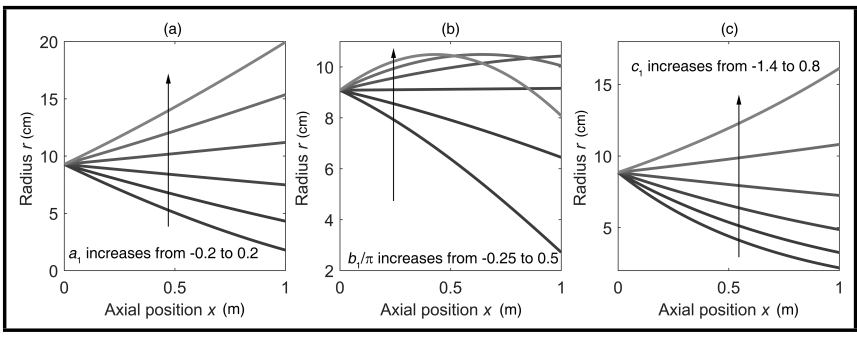

Figure 8. Duct radius changing with respect to geometrical parameters for (a) polynomial, (b) sinusoidal and (c) exponential ducts.

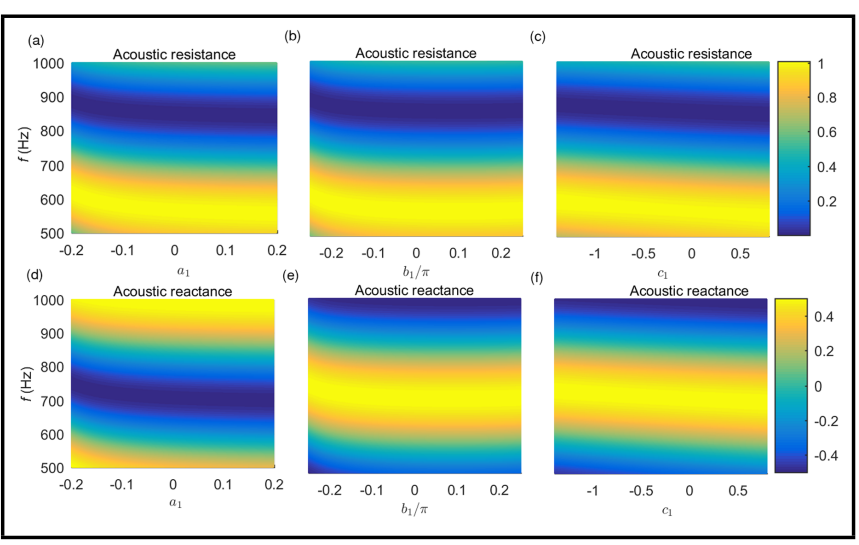

Figure 9. Changes of real $(a, b, c)$ and imaginary $(d, e, f)$ parts of the normalized side-branch impedances. (a), (d): polynomial; (b), (e): sinusoidal; and (c), (f): exponential ducts.

Figs. 8(a), (b) and (c), respectively, when $a_{1}$ ranges from -0.2 to $0.2 ; b_{1} / \pi$ ranges from -0.25 to 0.5 ; and $c_{1}$ ranges from -1.4 to 0.8 . From these plots, it is clear that for negative values of $a_{1}, b_{1}, c_{1}$, the diameters along the axial position of ducts are small enough so that only plane waves could propagate, but for positive values of these CSA parameters, higher-orders modes may exist.

The effects of the CSA variations and frequency on the sidebranch impedances are visible in Fig. 9, where the real and imaginary parts of the side-branch impedances for localization of traveling and standing waves are plotted against $a_{1}, b_{1} / \pi$, and $c_{1}$ for polynomial, sinusoidal and exponential ducts. It was seen that, for fixed excitation frequency and side-branch location, the real part of the side-branch impedance changes more than the imaginary part with respect to variations of duct CSA. This is because the change of duct area has more significant effects on the acoustic pressure amplitude than on the phase. For fixed duct CSA, the required acoustic impedances change almost sinusoidally with respect to the frequency. Though the change of the side-branch impedance is small with respect to the CSA parameters, the corrections were required because we have seen that the localization of traveling and standing waves was very sensitive to the impedances. ${ }^{2}$

The traveling-wave measure using side-branch impedances with and without corrections for CSA variation were compared using 3D FEA. The traveling-wave measures plotted against $a_{1}$, when the side-branch impedances were calculated 


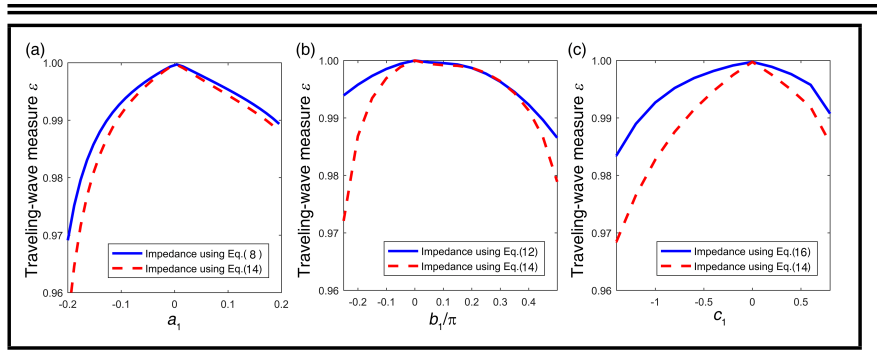

Figure 10. Traveling-wave measure plotted against the CSA parameters of the (a) polynomial, (b) sinusoidal and (c) exponential ducts using different sidebranch impedances. For reference, the result (red dashed line) obtained with the impedance given by Eq. (27), for a uniform duct, is shown in each case.

by Eqs. (17) and (27), are shown in Fig. 10(a). It was seen that the traveling-wave measure was maximum near $a_{1}=0$, where the duct CSA was constant, and decreased when the duct CSA was varying. This was because more reflections were generated due to the variation of acoustic impedance and a 3D wave effects if the duct CSA was varying more rapidly. ${ }^{20}$ The traveling-wave measure was not symmetric for ducts having a contraction and an expansion. The results showed that better localization of traveling and standing waves was obtained using the side-branch impedances with corrections for CSA variations. We also noticed that, in a duct with a radius larger than $10 \mathrm{~cm}$, higher-order acoustic modes exist. However, the localization of traveling and standing waves is not bad when $a_{1}, b_{1}$ and $c_{1}$ are positive numbers, as shown in Fig. 10.

The traveling-wave measure was plotted against $b_{1} / \pi$ (for the sinusoidal duct) and $c_{1}$ (for the exponential duct) in Figs. 10(b) and (c), respectively. Similar results were obtained as for the polynomial duct. Therefore, the conditions of sidebranch impedances obtained in this work were better approximations for the 3D polynomial, sinusoidal and exponential ducts.

\section{CONCLUDING REMARKS}

The exact solutions for side-branch impedances leading to spatial localization of traveling and standing waves in rigidwalled circular ducts with geometries of polynomial, sinusoidal, and exponential variations in the longitudinal dimension have been obtained analytically and verified numerically by 3D FEA. It was found that only small reflections in the left regions of the ducts were generated, and that these were due to variation of acoustic impedance and 3D wave effects. For all three geometrical configurations examined, the real part of the impedance was shown to be strictly positive over the space of duct parameters, side branch locations, and excitation frequencies, demonstrating that the proposed mathematical models were physically attainable.

The effects of CSA parameters on the required side-branch impedances have been studied. It was found that the real part of the side branch impedance changes more than the imaginary part with respect to variation of the duct CSA. Parametric studies showed that localization of traveling and standing waves using the side-branch impedances with corrections for CSA variation were better than those obtained without these corrections.

\section{ACKNOWLEDGEMENTS}

This research was supported by the National Natural Science Foundation of China through Grant No. 51975525, the Ministry of Science and Technology of the People's Republic of China through Grant No. 2017YFC0306202, the Department of Science and Technology of Zhejiang Province through Grant No. 2018C04018, and the Natural Science Foundation of Zhejiang Province through Grant No. LQ18E050012.

\section{REFERENCES}

1 Y. Xiao, A. Blanchard, Y. Zhang, H. Lu, D. M. McFarland, A. F. Vakakis and L. A. Bergman. Separation of traveling and standing waves in a rigid-walled circular duct containing an intermediate impedance discontinuity, J. Vib. Acoust. 139, 061001, (2017). https://dx.doi.org/10.1115/1.4036866

2 Y. Xiao, H. Lu, D. M. McFarland, A. F. Vakakis and L. A. Bergman. Inducing a nonreflective airborne discontinuity in a circular duct by using a nonresonant side branch to create mode complexity, J. Acoust. Soc. Am. 143(2), 746-755, (2018). https://dx.doi.org/10.1121/1.5022777

3 Y. Xiao, H. Lu, D. Zhou, D. M. McFarland, A. F. Vakakis and L. A. Bergman. Realization by impedance discontinuity of a unidirectional wave in a duct with harmonically perturbed uniform mean flow, J. Acoust. Soc. Am. 145(5), 3048-3057, (2019). https://dx.doi.org/10.1121/1.5108834

4 A. Blanchard, O. V. Gendelman, D. M. McFarland, L. A. Bergman and A. F. Vakakis. Mode complexity in a harmonically forced string with a local spring-damper and transitions from vibrations to waves, J. Sound Vib. 334, 282-295, (2015). https://dx.doi.org/10.1016/j.jsv.2014.08.033

5 A. Blanchard, D. M. McFarland, L. A. Bergman and A. F. Vakakis. Damping-induced interplay between vibrations and waves in a forced non-dispersive elastic continuum with asymmetrically placed local attachments, Proc. Roy. Soc. A-Math. Phy. 471(2176), 20140402, (2015). https://dx.doi.org/10.1098/rspa.2014.0402

6 X. Cheng, A. Blanchard, C. A. Tan, H. Lu, L. A. Bergman, D. M. McFarland and A. F. Vakakis. Separation of traveling and standing waves in a finite dispersive string with partial or continuous viscoelastic foundation, J. Sound Vib. 411, 193-209, (2017). https://dx.doi.org/10.1016/j.jsv.2017.08.028

7 X. Cheng, L. A. Bergman, D. M. McFarland, C. A. Tan, A. F. Vakakis, H. Lu. Co-existing complexity-induced traveling wave transmission and vibration localization in Euler-Bernoulli beams, J. Sound Vib. 458, 22-43, (2019). https://dx.doi.org/10.1016/j.jsv.2019.06.001

8 S. Motaharibidgoli, V. V. N. S. Malladi, P. A. Tarazaga. Developing a passive vibration absorber to generate traveling waves in a beam, in: N. Dervilis (Eds) Special Topics in Structural Dynamics, Volume 5, Conference Proceedings in the Society for Experimental Mechanics Series (Proceedings of the 36th IMAC, A Conference and Exposition on Structural Dynamics 2018), Springer, Cham, Switzerland, 2019, 245-248. https://dx.doi.org/10.1007/978-3319-75390-4_22

9 W. P. Mason. A barium titanate transducer capable of large motion at an ultrasonic frequency. J. Acoust. Soc. Am. 23(2), 209-214, (1951). https://dx.doi.org/10.1121/1.1906746 
10 M. Itoh, S. Tamano, K. Yokota. Drag reduction in a turbulent boundary layer on a flexible sheet undergoing a spanwise traveling wave motion, J. Turbul. 7(27), 1-17, (2006). https://dx.doi.org/10.1080/14685240600647064

11 H. Duifhuis. Cochlear Mechanics: Introduction to a Time Domain Analysis of the Nonlinear Cochlea, Springer, New York, (2012), Chap. 3, 33-39. https://dx.doi.org/10.1007/978-1-4419-6117-4

12 A. G. Webster. Acoustical impedance and the theory of horns and of the phonograph, Proc. Natl. Acad. Sci. USA. 5(7), 275-282, (1919). https://dx.doi.org/10.1073/pnas.5.7.275.

13 V. Salmon. Generalized plane wave horn theory, J. Acoust. Soc. Am. 17(6), 199-211, (1945). https://dx.doi.org/10.1121/1.1916316.

14 P. A. Martin. On Webster's horn equation and some generalizations, J. Acoust. Soc. Am. 116(3), 1381-1388, (2004). https://dx.doi.org/10.1121/1.1775272.

15 S. W. Rienstra. Webster's horn equation revisited, SIAM J. Appl. Math. 65(6), 1981-2004, (2005). https://dx.doi.org/10.1137/s0036139902413040.

16 V. Easwaran and M. L. Munjal. Plane wave analysis of conical and exponential pipes with incompressible mean flow, J. Sound Vib. 152(1), 73-93, (1992).

17 B. M. Kumar and R. I. Sujith. Exact solutions for the longitudinal vibration of non-Uniform rods, J. Sound Vib. 207(5), 721-729, (1997). https://dx.doi.org/10.1006/jsvi.1997.1146.

18 A. Cummings. Ducts with axial temperature gradients: an approximate solution for sound transmission and generation. J. Sound Vib. 51(1), 55-67, (1977). https://dx.doi.org/10.1016/S0022-460X(77)80112-0

19 E. Dokumaci. An approximate analytical solution for plane sound wave transmission in inhomogeneous ducts. J. Sound Vib. 217(5), 853-867, (1998). https://dx.doi.org/10.1006/jsvi.1998.1785

20 P. B. Subrahmanyam, R. I. Sujith, T. C. Lieuwen. A family of exact transient solutions for acoustic wave propagation in inhomogeneous, non-uniform area ducts. J. Sound Vib. 240(4), 705-715 (2001). https://dx.doi.org/10.1006/jsvi.2000.3261

21 I. Duran, S. Moreau. Solution of the quasi-one-dimensional linearized Euler equations using flow invariants and the Magnus expansion. J. Fluid Mech. 723, 190-231 (2013). https://dx.doi.org/10.1017/jfm.2013.118

22 S. R. Yeddula, A. S. Morgans. A semi-analytical solution for acoustic wave propagation in varying area ducts with mean flow. J. Sound Vib. 492, 115770, (2021). https://dx.doi.org/10.1016/j.jsv.2020.115770

23 L. Godinho, P. A. Mendes, J. Ramis, W. Cardenas and J. Carbajo. A numerical MFS model for computational analysis of acoustic horns, Acta Acust. United Ac. 98(6), 916927, (2012). https://dx.doi.org/10.3813/AAA.918575.
24 H. Thomas, T. Hézard, R. Mignot and D. Matignon. Onedimensional acoustic models of horns and comparison with measurements, Acta Acust. United Ac. 99(6), 960-974, (2013). https://dx.doi.org/10.3813/AAA.918675.

25 W. S. Rienstra. Sound propagation in slowly varying lined flow ducts of arbitrary crosssection, J. Fluid Mech. 495, 157-173, (2003). https://dx.doi.org/10.1017/S0022112003006050

26 M. L. Munjal. Acoustics of Ducts and Mufflers, John Wiley \& Sons, New York, 2nd ed., (2014), Chap. 1. pp. 1-39. ISBN: 978-1-118-44312-5

27 C. Q. Howard and B. S. Cazzolato. Acoustic Analyses Using Matlab ${ }^{\circledR}$ and Ansys ${ }^{\circledR}$ CRC Press, Boca Raton, (2014), Chap. 2, 52-53. https://dx.doi.org/10.1201/b17825

28 ANSYS ${ }^{\circledR}$ Academic Research, Release 15.0, Help System. ANSYS Mechanical APDL Modeling and Meshing Guide, ANSYS, SAS IP, Inc, (2013).

\section{APPENDIX A. DERIVATION OF Eq. (25)}

Introducing the transformation $\hat{q}_{i}(x)=\hat{p}_{i}(x) \sin \left(b_{1} x+b_{2}\right)$, $i=1,2$, Eq. (1) can be rewritten as: ${ }^{17}$

$$
\frac{\mathrm{d}^{2} \hat{q}_{i}(x)}{\mathrm{d} x^{2}}+k_{1}^{2} \hat{q}_{i}(x)=0 ;
$$

where $k_{1}^{2}=k^{2}+b_{1}^{2}$. Thus, the acoustic pressures in the two regions of the duct separated by the side branch are:

$$
\begin{array}{r}
p_{1}(x, t)=\left(C_{1} e^{-\mathrm{j} k_{1} x}+C_{2} e^{\mathrm{j} k_{1} x}\right) \csc \left(b_{1} x+b_{2}\right) e^{\mathrm{j} \Omega t}, \\
0 \leq x \leq x_{0} ; \\
p_{2}(x, t)=\left(D_{1} e^{-\mathrm{j} k_{1} x}+D_{2} e^{\mathrm{j} k_{1} x}\right) \csc \left(b_{1} x+b_{2}\right) e^{\mathrm{j} \Omega t}, \\
x_{0} \leq x \leq L .
\end{array}
$$

The acoustic particle velocities $u_{1}(x, t)$ and $u_{2}(x, t)$ in the two regions of the duct are determined by Eq. (6). The boundary conditions are identical to those of the previous problem and lead to solutions for the unknown coefficients $C_{1}, C_{2}, D_{1}$, and $D_{2}$, which are found to be:

$$
\begin{aligned}
& C_{1}=-\frac{F \sin \left(b_{2}\right) e^{2 \mathrm{j} k_{1} x_{0}}}{G_{2}} \\
& \cdot\left[k_{1}\left(\left(2 Z_{0}+1\right) e^{2 \mathrm{j} k_{1} L}+e^{2 \mathrm{j} k_{1} x_{0}}\right)\right. \\
& +\left(\mathrm{j} b_{1} \cot \left(b_{1} L+b_{2}\right)\left(\left(2 Z_{0}+1\right) e^{2 \mathrm{j} k_{1} L}-e^{2 \mathrm{j} k_{1} x_{0}}\right)\right] ;
\end{aligned}
$$

$$
\begin{gathered}
C_{2}=\frac{F \sin \left(b_{2}\right)}{G_{2}}\left[k_{1}\left(e^{2 \mathrm{j} k_{1} L}+\left(1-2 Z_{0}\right) e^{2 \mathrm{j} k_{1} x_{0}}\right)\right. \\
\left.+\mathrm{J} b_{1} \cot \left(b_{1} L+b_{2}\right)\left(e^{2 \mathrm{j} k_{1} L}+\left(2 Z_{0}-1\right) e^{2 \mathrm{j} k_{1} x_{0}}\right)\right] ;
\end{gathered}
$$

$$
D_{1}=-\frac{2 F \sin \left(b_{2}\right) Z_{0} e^{2 \mathrm{j} k_{1}\left(L+x_{0}\right)}}{G_{2}} \cdot\left(k_{1}+\mathrm{j} b_{1} \cot \left(b_{1} L+b_{2}\right)\right) ;
$$




$$
\begin{aligned}
D_{2}=-\frac{2 F \sin \left(b_{2}\right) Z_{0} e^{2 \mathrm{j} k_{1} x_{0}}}{G_{2}} \cdot\left(k_{1}-\mathrm{j} b_{1} \cot \left(b_{1} L+b_{2}\right)\right) ;
\end{aligned}
$$

where:

$$
\begin{aligned}
& G_{2}= \mathrm{j} b_{1} \cot \left(b_{1} L+b_{2}\right) \\
&\left(e^{2 \mathrm{j} k_{1} L}+e^{4 \mathrm{j} k_{1} x_{0}}-\left(2 Z_{0}+1\right) e^{2 \mathrm{j} k_{1}\left(L+x_{0}\right)}\right. \\
&\left.+\left(2 Z_{0}-1\right) e^{2 \mathrm{j} k_{1} x_{0}}\right) \\
&+k_{1}\left(e^{2 \mathrm{j} k_{1} L}-e^{4 \mathrm{j} k_{1} x_{0}}-\left(2 Z_{0}+1\right) e^{2 \mathrm{j} k_{1}\left(L+x_{0}\right)}\right. \\
&\left.-\left(2 Z_{0}-1\right) e^{2 \mathrm{j} k_{1} x_{0}}\right) .
\end{aligned}
$$

Equation (25) is obtained by setting to zero the coefficient $C_{2}$ of the reflected wave in the left region.

Substituting Eq. (25) into Eqs. (35)-(39) gives:

$$
\begin{gathered}
C_{1}=F \sin \left(b_{2}\right) \\
C_{2}=0 \\
D_{1}=\frac{F \sin \left(b_{2}\right)}{2} \\
\cdot\left[1+\frac{\mathrm{j}}{E_{2}^{2}}\left(2 b_{1} k_{1} \sin \left(2\left(b_{1} L+b_{2}\right)\right) \cos \left(2 k_{1}\left(L-x_{0}\right)\right)\right.\right. \\
\left.\left.\cdot\left(\left(b_{1}^{2}+k_{1}^{2}\right) \cos \left(2\left(b_{1} L+b_{2}\right)\right)+b_{1}^{2}-k_{1}^{2}\right)\right)\right] \\
D_{2}=\frac{F \sin \left(b_{2}\right)}{\frac{\mathrm{e}^{2 \mathrm{j} k_{1} L}\left(k_{1}+\mathrm{j} b_{1} \cot \left(b_{1} L+b_{2}\right)\right)}{k_{1}-\mathrm{j} b_{1} \cot \left(b_{1} L+b_{2}\right)}+\mathrm{e}^{2 \mathrm{j} k_{1} x_{0}}} ;
\end{gathered}
$$

where:

$$
\begin{aligned}
E_{2}=\left(b_{1}-k_{1}\right) \sin \left(b_{1} L+b_{2}+k_{1}\left(L-x_{0}\right)\right) \\
\quad+\left(b_{1}+k_{1}\right) \sin \left(b_{1} L+b_{2}-k_{1}\left(L-x_{0}\right)\right) .
\end{aligned}
$$

Substituting Eqs. (40) and (41) into Eq. (33), it is clear that a traveling wave is observed in the left region of the duct because $C_{2}=0$. However, caution should be taken when $E_{2}=0$ and $D_{1}=D_{2}=\infty$, corresponding to resonance in the right region of duct. The existence of this singularity implies that Eq. (25) is a necessary, but not sufficient, condition for realization of a traveling wave.

\section{APPENDIX B. DERIVATION OF Eq. (29)}

Substituting Eq. (28) into Eq. (1), we obtain:

$$
\frac{\mathrm{d}^{2} \hat{p}(x)}{\mathrm{d} x^{2}}+2 c_{1} \frac{\mathrm{d} \hat{p}(x)}{\mathrm{d} x}+k^{2} \hat{p}(x)=0 .
$$

Further substituting the assumption $\hat{p}(x)=e^{\mathrm{j}_{3} x}$ into Eq. (45) gives $k^{2}-k_{3}^{2}+2 \mathrm{\jmath} c_{1} k_{3}=0$, from which:

$$
k_{3}=\mathrm{j} c_{1} \pm \sqrt{k^{2}-c_{1}^{2}}=\mathrm{j} c_{1} \pm k_{2}
$$

The acoustic pressures in the two regions of the duct separated by the side branch are:

$$
\begin{aligned}
p_{1}(x, t)=e^{-c_{1} x}\left(C_{1} e^{-\mathrm{j} k_{2} x}+C_{2} e^{\mathrm{j} k_{2} x}\right) e^{\mathrm{j} \Omega t} & , \\
& 0 \leq x \leq x_{0}
\end{aligned}
$$

$$
\begin{aligned}
p_{2}(x, t)=e^{-c_{1} x}\left(D_{1} e^{-\mathrm{j} k_{2} x}+D_{2} e^{\mathrm{j} k_{2} x}\right) e^{\mathrm{j} \Omega t}, & \\
x_{0} & \leq x \leq L ;
\end{aligned}
$$

and the acoustic particle velocities $u_{1}(x, t)$ and $u_{2}(x, t)$ are determined by Eq. (6). The boundary conditions are consistent with those of the previous two problems and lead to solutions for the unknown coefficients $C_{1}, C_{2}, D_{1}$, and $D_{2}$, of the form:

$$
\begin{aligned}
& C_{1}=\left(2 \mathrm{e}^{2 \mathrm{j} k_{2} L} Z_{0} k_{2}^{2}+j\left(\mathrm{e}^{2 \mathrm{j} k_{2} L}-\mathrm{e}^{2 \mathrm{j} k_{2} x_{0}}\right) k c_{1}\right. \\
& \left.+\left(\left(\mathrm{e}^{2 \mathrm{j} k_{2} L}+\mathrm{e}^{2 \mathrm{j} k_{2} x_{0}}\right) k+2 \mathrm{je}^{2 \mathrm{j} k_{2} L} c_{1} Z_{0}\right) k_{2}\right) \times \frac{F \mathrm{e}^{2 \mathrm{j} k_{2} x_{0}}}{G_{3}}
\end{aligned}
$$

$$
\begin{gathered}
C_{2}=\left(2 \mathrm{e}^{2 \mathrm{j} k_{2} x_{0}} Z_{0} k_{2}^{2}-\mathrm{j}\left(\mathrm{e}^{2 \mathrm{j} L k_{2}}-\mathrm{e}^{2 \mathrm{j} k_{2} x_{0}}\right) k c_{1}\right. \\
\left.-\left(\left(\mathrm{e}^{2 \mathrm{j} L k_{2}}+\mathrm{e}^{2 \mathrm{j} k_{2} x_{0}}\right) k+2 \mathrm{je} \mathrm{j}^{2 \mathrm{j} k_{2} x_{0}} c_{1} Z_{0}\right) k_{2}\right) \times \frac{F}{G_{3}} \\
D_{1}=\frac{2 F}{G_{3}} k_{2} Z_{0}\left(k_{2}+\mathrm{j} c_{1}\right) \mathrm{e}^{2 \mathrm{j} k_{2}\left(L+x_{0}\right)} ; \\
D_{2}=\frac{2 F}{G_{3}} k_{2} Z_{0}\left(k_{2}-\mathrm{j} c_{1}\right) \mathrm{e}^{2 \mathrm{j} k_{2} x_{0}}
\end{gathered}
$$

where:

$$
\begin{gathered}
G_{3}=\left(\left(\mathrm{e}^{2 \mathrm{j} k_{2} x_{0}}-1\right)\left(\mathrm{e}^{2 \mathrm{j} k_{2} L}+\mathrm{e}^{2 \mathrm{j} k_{2} x_{0}}\right) k\right. \\
\left.+2 \mathrm{j} \mathrm{e}^{2 \mathrm{j} k_{2} x_{0}}\left(\mathrm{e}^{2 \mathrm{j} k_{2} L}-1\right) c_{1} Z_{0}\right) k_{2} \\
+2 \mathrm{e}^{2 \mathrm{j} k_{2} x_{0}}\left(1+\mathrm{e}^{2 \mathrm{j} k_{2} L}\right) Z_{0} k_{2}^{2} \\
+\mathrm{j}\left(\mathrm{e}^{2 \mathrm{j} k_{2} L}-\mathrm{e}^{2 \mathrm{j} k_{2} x_{0}}\right)\left(\mathrm{e}^{2 \mathrm{j} k_{2} x_{0}}-1\right) k c_{1} .
\end{gathered}
$$

Equation. (29) is obtained by setting the coefficient of the reflected wave in the left region to zero, $C_{2}=0$.

Substituting Eq. (29) into (49)-(52) gives:

$$
\begin{aligned}
& C_{1}=F ; \\
& C_{2}=0 ;
\end{aligned}
$$

$$
\begin{aligned}
& D_{1}= \\
& \frac{F}{2}\left(1-\mathrm{j} \frac{c_{1} \cos \left(k_{2}\left(L-x_{0}\right)\right)+k_{2} \sin \left(k_{2}\left(L-x_{0}\right)\right)}{c_{1} \sin \left(k_{2}\left(L-x_{0}\right)\right)-k_{2} \cos \left(k_{2}\left(L-x_{0}\right)\right)}\right) ;
\end{aligned}
$$

$$
\begin{aligned}
& D_{2}= \\
& \quad-F \frac{c_{1}+\mathrm{j} k_{2}}{c_{1}\left(\mathrm{e}^{2 \mathrm{j} k_{2} L}-\mathrm{e}^{2 \mathrm{j} k_{2} x_{0}}\right)-\mathrm{j} k_{2}\left(\mathrm{e}^{2 \mathrm{j} k_{2} L}+\mathrm{e}^{2 \mathrm{j} k_{2} x_{0}}\right)}
\end{aligned}
$$

and substituting Eqs. (54) and (55) into Eq. (47), it is clear that a traveling wave is confined to the left region of the duct. However, when the conditions $c_{1}=k_{2} \cot \left(k_{2}\left(L-x_{0}\right)\right)$, $D_{1}=D_{2}=\infty$, hold, this corresponds to resonance in the right region of duct. The existence of this singularity implies that Eq. (29) is a necessary, but not sufficient, condition for realization of a traveling wave. 